\title{
(Meta-)stability and Core-Shell Dynamics of Gold Nanoclusters at Finite Temperature
}

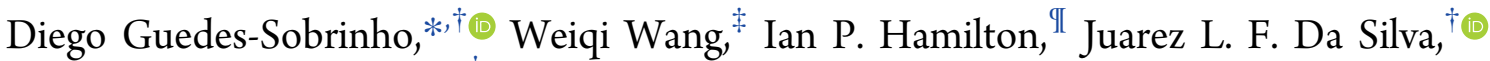 \\ and Luca M. Ghiringhelli**; \\ ${ }^{\dagger}$ São Carlos Institute of Chemistry, University of São Paulo, PO Box 780, 13560-970 São Carlos, São Paulo, Brazil \\ ${ }^{\ddagger}$ Fritz-Haber-Institut der Max-Planck-Gesellschaft, 14195 Berlin-Dahlem, Germany \\ ${ }^{\mathbb{I}}$ Department of Chemistry and Biochemistry, Wilfrid Laurier University, Waterloo N2L 3C5, Ontario, Canada
}

Supporting Information

\begin{abstract}
Gold nanoclusters have been the focus of numerous computational studies, but an atomistic understanding of their structural and dynamical properties at finite temperature is far from satisfactory. To address this deficiency, we investigate gold nanoclusters via $a b$ initio molecular dynamics, in a range of sizes where a core-shell morphology is observed. We analyze their structure and dynamics using state-of-the-art techniques, including unsupervised machine-learning nonlinear dimensionality reduction (sketch-map) for describing the similarities and differences among the range of sampled configurations. In the examined temperature range between 300 and $600 \mathrm{~K}$, we find that whereas the gold nanoclusters exhibit continuous structural rearrangement, they are not amorphous. Instead, they clearly show persistent motifs: a cationic core of $1-5$ atoms is loosely bound to a shell which typically displays a substructure resulting from the competition between locally spherical versus planar fragments. Besides illuminating the properties of core-shell gold nanoclusters, the present study proposes a set of useful tools for understanding their nature in operando.
\end{abstract}

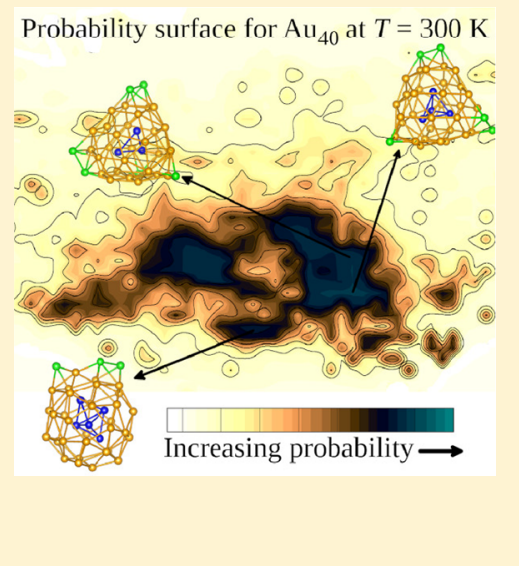

$\mathrm{T}$ ransition-metal nanoclusters have been the focus of a large number of experimental and computational studies in physics and chemistry because of their expected technological applications in diverse areas, including catalysis, ${ }^{1}$ optics, ${ }^{2}$ and biomedicine. ${ }^{3}$ Their physical and chemical properties are dependent on many characteristics such as size, ${ }^{4}$ shape, ${ }^{5}$ charge state, ${ }^{6}$ ligand effects, ${ }^{7}$ and temperature. ${ }^{8}$ Transition-metal nanoclusters typically exhibit catalytic activity at high temperature, ${ }^{9}$ but gold nanoclusters have recently received special attention because of the discovery of their catalytic activity at low temperature. ${ }^{10}$

Experimental and computational studies of both colloi$\mathrm{dal}^{11-15}$ and bare ${ }^{16-23}$ gold nanoclusters have achieved important advances in the last decades. Most of the reported $a b$ initio studies have been dedicated to searching for their putative global (energy) minimum configurations (pGMC) in the gas phase at zero temperature. ${ }^{14}$ An empirical-potential molecular dynamics (MD) study of $\mathrm{Au}_{75}, \mathrm{Au}_{146}$, and $\mathrm{Au}_{457}$ showed that their melting temperatures were well below the melting temperature of bulk gold $(1090 \mathrm{~K}$, at standard pressure) but also that structural solid-to-solid transitions were present well below their melting temperatures. ${ }^{24}$ For instance, for $\mathrm{Au}_{75}$, the melting temperature is around $550 \mathrm{~K}$, but around $350 \mathrm{~K}$ there is a structural transition from truncated-decahedral to icosahedral.

The present study focuses on the gas-phase gold nanoclusters $\mathrm{Au}_{25}, \mathrm{Au}_{38}$, and $\mathrm{Au}_{40} . \mathrm{Au}_{25}$ was chosen because it is expected to be the smallest cluster that has a core, in this case a single $\mathrm{Au}$ atom, in the low-lying isomer population. ${ }^{18} \mathrm{Au}_{38}$ and $\mathrm{Au}_{40}$ were chosen because they are both expected to have tetrahedral $\mathrm{Au}_{4}$ cores and low-symmetry structures have been proposed as their pGMCs. ${ }^{22,23}$ We use ab initio BornOppenheimer $\mathrm{MD}$, an important computational technique that allows for the sampling of the configurational space of systems with many atoms, together with yielding reliable information on the dynamics of the system, for example, in terms of timecorrelation functions. When the Born-Oppenheimer MD equations of motion are extended by adding a thermostat, the canonical ensemble (at temperature $T>0 \mathrm{~K}$ ) is sampled. In the specific case of density-functional theory (DFT)-based $\mathrm{MD}$, the energy of the system and forces between atoms are determined by solving the Kohn-Sham equations. Hence, the ground-state electronic structure is accessible along the simulated trajectories.

DFT-based MD studies on small $\mathrm{Au}$ clusters have been performed in the past, aimed at describing the thermal evolution of the ground-state structures and the competition between structural isomers at a given temperature, ${ }^{25-27}$ as well as at accessing the infrared (vibrational) spectra for comparison with experiments. ${ }^{28}$ We analyze DFT-based MD trajectories of $\mathrm{Au}_{25}, \mathrm{Au}_{38}$, and $\mathrm{Au}_{40}$ at room and higher

Received: November 7, 2018

Accepted: January 25, 2019

Published: January 25, 2019 


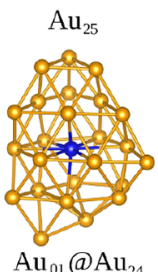

$0.00 \mathrm{eV}$

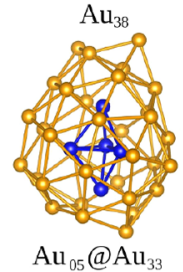

$0.72 \mathrm{eV}$

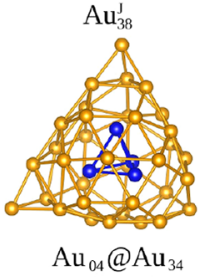

$0.00 \mathrm{eV}$
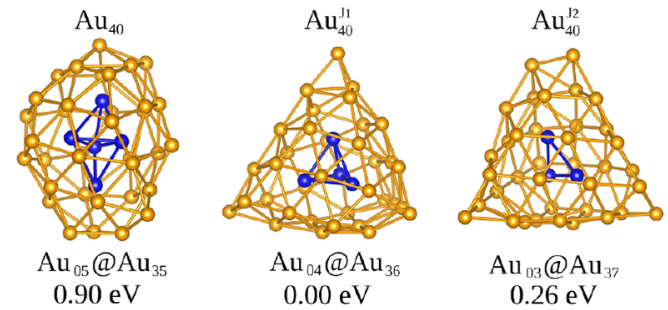

Figure 1. Relative total energies and configurations used as starting points of the MD trajectories. Structures labeled as $\mathrm{Au}_{38}^{\mathrm{J}}, \mathrm{Au}_{40}^{\mathrm{J}}$, and $\mathrm{Au}_{40}^{\mathrm{J}}$ are from ref 19, while structures labeled as $\mathrm{Au}_{25}, \mathrm{Au}_{38}$, and $\mathrm{Au}_{40}$ are found via EAM-RBHMC and further optimized with PBE+MBD (see text for details). The core (shell) atoms are indicated by blue (yellow). The labeling $\mathrm{Au}_{\mathrm{c}} @ \mathrm{Au}_{\mathrm{s}}$ marks the number of atoms in the core (c) and shell (s).
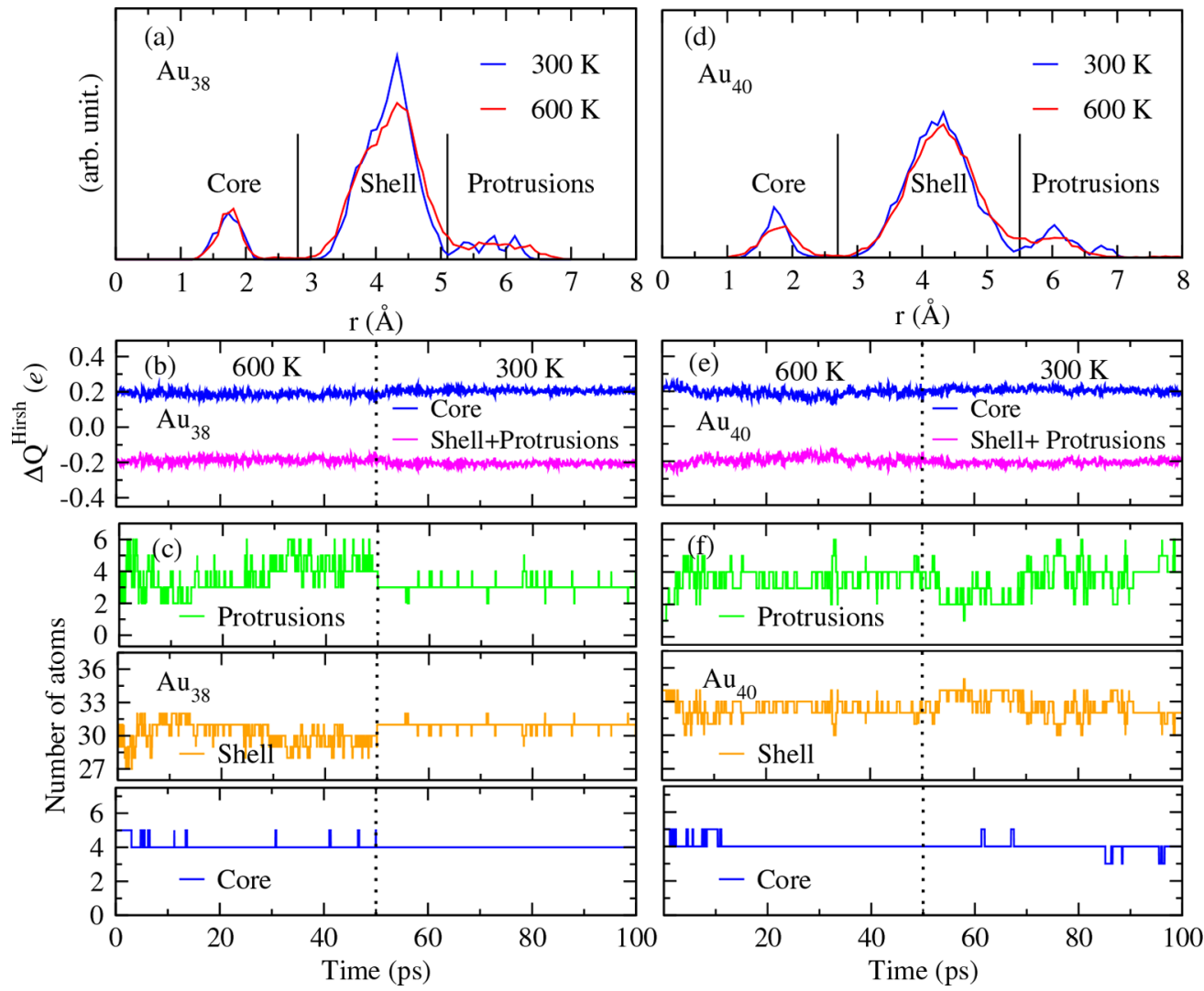

Figure 2. (a) Radial distribution function, $g(r)$, and cutoff distances (solid vertical lines) with respect to the cluster's center of mass defining the core, shell, and protrusion regions for the $\mathrm{Au}_{38}$ nanocluster at 600 and $300 \mathrm{~K}$. (b) Evolution of the Hirshfeld charges for core and shell atoms and (c) evolution of number of atoms belonging to protrusions (green line), shell (orange line), and core (blue line) regions at 600 and $300 \mathrm{~K}$ for $\mathrm{Au}_{38}$. Panels $\mathrm{d}-\mathrm{f}$ show the same quantities as in panels $\mathrm{a}-\mathrm{c}$, respectively, but for $\mathrm{Au}_{40}$.

temperatures in order to address important questions regarding the structural and dynamical aspects specific to this range of cluster sizes: (i) Is the core-shell morphology persistent at finite temperature? (ii) Do the atoms constituting the core exchange their positions with those constituting the shell? (iii) Does the shell of different isomers have recurrent structural motifs?

We ran DFT-based MD within the Perdew-BurkeErnzerhof $^{29}$ (PBE) approximation for the electronic exchange-correlation functional, corrected for the many-body dispersion (MBD) interactions, ${ }^{30}$ in the all-electron fullpotential implementation of the FHI-AIMS package. ${ }^{31}$ The time step for the numerical integration of the MD equations of motion was set to $10 \mathrm{fs}$, and we applied a Gaussian broadening of $1 \mathrm{meV}$ for the occupation at the Fermi level. Canonical sampling was imposed via the stochastic velocity-rescaling thermostat. $^{32}$ Further MD details are described in the
Supporting Information. These settings have been already adopted in refs 27, 28, and 33 and were shown to yield observable quantities in good agreement with experiments in the size range between 3 and 20 gold atoms. Gold nanoclusters are strongly influenced by relativistic effects, ${ }^{34,35}$ as the contraction of the $6 \mathrm{~s}$ orbital and expansion of the $5 \mathrm{~d}$ results in a smaller $\mathrm{s}-\mathrm{d}$ gap and an increased directionality in the $\mathrm{Au}-$ $\mathrm{Au}$ bonding. In the present study, relativistic effects are included via the "atomic" zeroth-order regular approximation to the Dirac equation. ${ }^{36}$

The initial configurations for the MD trajectory were (Figure 1) the pGMC structures described in ref 19 for $\mathrm{Au}_{38}$ and $\mathrm{Au}_{40}$, both containing a tetrahedral $\mathrm{Au}_{4}$ core, and a higher-energy isomer for $\mathrm{Au}_{40}$ (also from ref 19), containing a $\mathrm{Au}_{3}$ core. Furthermore, to increase diversity in the starting point of the MD trajectories of ${A u_{38}}_{3}$ and $\mathrm{Au}_{40}$, we also selected the respective $\mathrm{pGMC}$ of a potential-energy surface described 
through the embedded-atom method (EAM), globally optimized via the revised basin-hopping Monte Carlo (RBHMC) algorithm, ${ }^{37}$ both containing a $\mathrm{Au}_{5}$ core. For $\mathrm{Au}_{25}$, we adopted the embedded-atom-RBHMC pGMC, which has a one-atom core. More details on the RBHMC search are given in Figure S1 of the Supporting Information. We note that close-packed structures, such as icosahedra and decahedra, which can be important for larger gold nanoclusters, are not relevant (i.e., energetically very unfavored) for the smaller sizes considered in the present Letter. ${ }^{38}$ For each starting point, one or more $a b$ initio MD trajectories of 100 ps is run, where the first interval of $50 \mathrm{ps}$ is thermostated at a temperature between 400 and $600 \mathrm{~K}$ (see Table S1 for details on the single trajectories) and the last interval of $50 \mathrm{ps}$ is at "room temperature", i.e., $300 \mathrm{~K}$.

As detailed below, the statistical analysis of the structure and dynamics of the studied clusters is performed by means of radial-distribution functions, bond-lifetime analysis, nonlinear dimensionality reduction (in order to represent, by exploiting "structure similarities", the high-dimensional configurational space in two dimensions), and state-of-the-art Boltzmann reweighting (in order to use the $\mathrm{MD}$ sampling at all temperatures in the most efficient way).

The (center-of-mass centered) radial-distribution function $g(r)$ is the histogram in which bin $i$ counts how many atoms are found between distance $r_{i-1}$ and $r_{i}$ from the center of mass of the cluster, averaged over all configurations along a trajectory. Here, $r_{0}=0$ and the radial increment $r_{i+1}-r_{i}=$ $\delta r$ is constant and set to $0.1 \AA$. For extended systems, the histogram is typically normalized by the volume of the spherical shell between $r_{i+1}$ and $r_{i}$, but for finite systems, this normalization is not relevant and omitted here. This descriptor, invariant with respect to rigid rotations and translations of the whole cluster and to permutations of atom identities, gives direct and statistical access to the radially layered structure of the clusters and allows for a parameter-free definition of the core and shell regions. Figure $2 \mathrm{a}$ shows representative $g(r)$ plots for one trajectory of $\mathrm{Au}_{38}$ and one trajectory of $\mathrm{Au}_{40}$. At both cluster sizes, three clear regions can be distinguished. Some gold atoms are found in the range 1.0$2.5 \AA$. These are the atoms constituting the core. Another group of atoms is found in the range 2.5-5.5 $\AA$. These are the atoms constituting the shell. A third group of atoms appears at larger distances. A minimum in $g(r)$ at $r \approx 5.1 \AA\left(\mathrm{Au}_{38}\right)$ and $r$ $\approx 5.5 \AA\left(\mathrm{Au}_{40}\right)$ suggests that atoms contributing to the outer part of the distribution form a well-separated group from the shell atoms. Visual inspection of the cluster structures (e.g., green-colored spheres in Figure 4) reveals that these are lowercoordinated atoms "protruding" from an otherwise triangularlattice shell, the latter typically composed of 6-7 fold coordinated atoms. We therefore name this outer region protrusions. The $g(r)$ plots for all trajectories, classified also by temperature, are shown in Figures S2-S4. For $\mathrm{Au}_{38}$ and $\mathrm{Au}_{40}$, the three-region feature is observed for all trajectories and temperatures, with cutoff radii at $2.5 \AA$ for core-shell and in the range of 5.0-5.5 $\AA$ for shell-protrusions. The average numbers of gold atoms in the core/shell/protrusion regions (obtained by integrating the $g(r)$ in panels a and $\mathrm{d}$, which is the same as taking the time average of the trajectories in panels $\mathrm{c}$ and $\mathrm{f}$ ) are, for $\mathrm{Au}_{38}$ at $300 \mathrm{~K}(600), 4.0 / 31.0 / 3.0$ (4.1/30.2/ 3.7); for $\mathrm{Au}_{40}$ at $300 \mathrm{~K}(600), 4.0 / 31.8 / 4.2(4.1 / 32.2 / 3.6)$. We notice that for $\mathrm{Au}_{38}$, atoms move on average from protrusion to shell region when going from 600 to $300 \mathrm{~K}$, while the opposite trend is observed for $\mathrm{Au}_{40}$. The numbers of atoms in the core tends to remain constant. These temperature effects are accompanied by a less clear boundary between shell and protrusion regions for both cluster sizes at $600 \mathrm{~K}$, compared to $300 \mathrm{~K}$, signaled by a less marked minimum of $g(r)$ at $r \approx 5.5 \AA$. Another observation, from the trajectories in panels $\mathrm{c}$ and $\mathrm{f}$, is that both shell and protrusion regions for $\mathrm{Au}_{38}$ are rather stiff at $300 \mathrm{~K}$ while more dynamical at $600 \mathrm{~K}$ and for $\mathrm{Au}_{40}$ at both temperatures. For $\mathrm{Au}_{25}$, only a (one-atom) core region and a shell region (the latter in the range of $2.5-5.5 \AA$, as for the larger clusters) is observed.

We note here that atoms are observed to change region along the MD trajectories, so their assignment to a region is dynamical and based on solely the distance from the center of mass. This flexible definition is crucial for our subsequent analysis. In Figure 2, we also show the time evolution of the Hirshfeld charges ${ }^{39}$ of the core and shell+protrusion regions (panels $\mathrm{b}$ and $\mathrm{e}$ ), and of the number of atoms belonging to these regions (panels $c$ and $\mathrm{f}$ ), for $\mathrm{Au}_{38}$ and $\mathrm{Au}_{40}$. These observables are reported for all trajectories in Figures S2-S5. The qualitative behavior is remarkably consistent at all cluster sizes and temperatures: the core is always fractionally cationic, while the shell+protrusions is always fractionally anionic.

The number of atoms in the core is very stable. For $\mathrm{Au}_{25}$, it is always one, and for $\mathrm{Au}_{38}$, it is almost always four, with infrequent fluctuation to five (in the trajectories that started from the structure with a five-atom core, one atom quickly, in a few picoseconds, moves to the shell region). For $\mathrm{Au}_{40}$, the behavior is more complex. Trajectories starting with four atoms in the core maintain a four-atom core (with sporadic fluctuations to five) throughout the time evolution, even at the higher temperature $(600 \mathrm{~K})$. However, the trajectory starting from a three-atom core (structure "J2" in Figure 1) fluctuates between three and four atoms in the core for the 50 ps at 600 $\mathrm{K}$ and remains with a three-atom core during the subsequent $50 \mathrm{ps}$ time interval at $300 \mathrm{~K}$, indicating a kinetic trapping in a metastable state. When starting with a five-atom core (EAMRBHMC pGMC structure), it takes $500 \mathrm{~K}$ and more than 10 ps to see the trigonal-bipyramidal five-atom core transform into a tetrahedral four-atom core. At $600 \mathrm{~K}$, the transformation is faster (fraction of a picosecond, but followed by frequent fluctuation back to a five-atom core), while at $400 \mathrm{~K}$, the fiveatom core remains stable. The subsequent parts of the trajectories at $300 \mathrm{~K}$ maintain the number of atoms in the core, as at the higher temperature. In this case, we have also the signature of a kinetic trapping that is easily overcome at $600 \mathrm{~K}$.

The number of atoms belonging to either shell or protrusions regions (for $\mathrm{Au}_{38}$ and $\mathrm{Au}_{40}$ ) is quite stable, but with larger fluctuations $( \pm 2-3$ atoms around the average value along the trajectories). Average sizes of the protrusions regions range from 2 to 7 atoms, depending on the cluster size and the temperature.

To further analyze the nature of the different regions in the clusters, we measured the average bond lifetimes, according to whether the bonded pair belongs to one region or is across regions. To this purpose, we first define a bonded pair (at a given time step) as any pair of atoms at a distance smaller than 3.6 A. This cutoff value is assigned as the first minimum of the pair-distribution function. It is a concept similar to the $g(r)$ defined above, but here the distance between any pair of atoms is binned. The position of the first minimum (see Figure S6) is relatively independent of cluster size, temperature, and region 
to which the atoms belong. Next, if a bond is found at any time step, it is monitored until it is first broken (i.e., until the distance between the two atoms exceeds the threshold): The elapsed time is the bond lifetime, and it is binned considering whether both atoms are in the core, or one in the core and one in the shell, etc. In Figure 3, we show $\tau$, the region-dependent

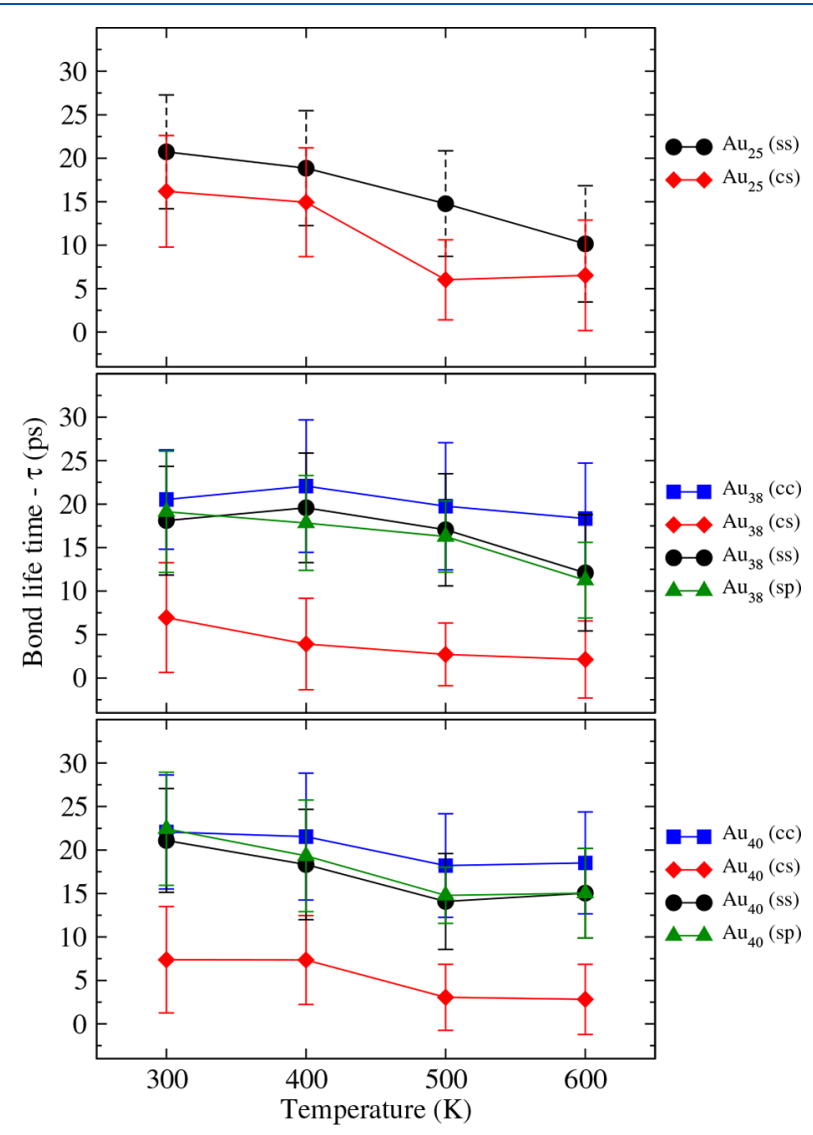

Figure 3. (a) Average bond lifetime, $\tau$, and sample standard deviation (bars) among the core-core atoms $\left(\tau_{\mathrm{cc}}\right)$, core-shell atoms $\left(\tau_{\mathrm{cs}}\right)$, shell-shell atoms $\left(\tau_{\mathrm{ss}}\right)$, and shell-protrusions $\left(\tau_{\mathrm{ps}}\right)$ atoms, for the $\mathrm{Au}_{25}$, $\mathrm{Au}_{38}$, and $\mathrm{Au}_{40}$ nanoclusters as a function of the sampled temperatures. The lines are a guide to the eye.

average value of the bond lifetime, at each cluster size and temperature (if more trajectories sampled the same temperature, we averaged over them). We also show the sample standard deviation of each bond-lifetime histogram as an error bar in order to quantify the spread of this quantity under the different conditions. More details on the bond lifetimes, broken down to single trajectories, are given in Table S2. We observe a striking difference in behavior between bonds shared by atoms both belonging to the core, or both belonging to the shell, and bonds across core and shell. The latter are systematically at least 3-4 times shorter lived than the former. The shell-protrusion bonds behave similarly to shell-shell bonds, suggesting that the two regions are structurally separated but dynamically very similar. Protrusion-protrusion bonds were too few to yield robust statistics at all sizes and temperatures. The dynamics of the core-shell bonds suggests a picture of a shell that is loosely bound to the core. Visual inspection of the trajectories of $\mathrm{Au}_{40}$ and $\mathrm{Au}_{38}$ confirms that the core atoms behave like a inner cluster "confined", and almost freely rotating, inside the shell. In the case of $\mathrm{Au}_{25}$, the single-atom core "rattles" around the center of mass.
Consistently, $g(r)$ for Au25 (Figure S3) shows that the oneatom core is positioned on average at about $0.25 \AA$ from the center of mass.

In order to describe the statistical distribution of structures at each cluster size, as sampled along the MD trajectories, we adopted an unsupervised machine-learning approach, namely, the nonlinear dimensionality-reduction algorithm termed sketch-map, as introduced in ref 40 . First, similarly to ref 41 , we describe each configuration along a MD trajectory with a coordination histogram, where the $j$ th bin is the number of atoms that have coordination $j$, i.e., that have $j$ atoms at a distance within the same threshold defined above for the bond breaking. A graphical example of how a cluster structure is mapped into a coordination histogram is given in Figure S7. The histogram is normalized; that is, each bin is divided by the number of atoms in the cluster. For the clusters considered, we found that the maximum coordination is 12 , and each histogram was therefore set to have dimension 13, starting from 0 -fold coordinated atoms (i.e., atoms evaporated from the clusters) which were, however, not observed in the present study. This 13-dimensional representation is already a dimensionality reduction from the $3 N$ coordinates for an $N$ atom cluster. Such representation is roto-translational and permutation invariant, similar to $g(r)$. In this preliminary dimensionality reduction, some information is necessarily lost, as two different clusters may accidentally have the same coordination histogram. Nonetheless, the coordination histogram captures essential information regarding the bonding network for the clusters. Next, we define a distance in the coordination-histogram representation, which is simply the Euclidean distance between histograms, considered as vectors. The sketch-map algorithm ${ }^{40}$ then embeds, in two dimensions, the high-dimensional points (here, in the coordinationhistogram representation), by representing points that are close (distant) in high dimension as close (distant) in the twodimensional (2-D) map. The axes in the plots do not have any physical meaning and cannot even be labeled explicitly; what matters are the distances. Still, each high-dimensional point is mapped into a specific point on the 2-D map. Therefore, by discretizing the 2-D coordinates, one can count how many points land in each discrete (2-D) bin and color-code the map according to the number of points in each bin. For better statistics, we adopted a Boltzmann reweighting approach, the multistate Bennet acceptance-ratio (MBAR) algorithm, ${ }^{42}$ to estimate the probability that a structure falls in a given bin, by using the trajectories at all temperatures, while the shown plot refers to $300 \mathrm{~K}$ only. In this way, the above-described kinetictrapping issues at $300 \mathrm{~K}$ are mitigated by simultaneous use of trajectories at higher temperatures, where no kinetic trapping is observed. For a converged sampling, the logarithm of these probabilities is proportional to the negative of the free-energy of the given $\mathrm{Au}_{N}$ cluster. In our case, however, the samplings are most likely not converged, and the probabilities are therefore only qualitative. The results for $\mathrm{Au}_{38}$ and $\mathrm{Au}_{40}$ are shown in Figure 4 (results for $\mathrm{Au}_{25}$ are in Figure S8). Absolute errors for the same sketch-maps, also evaluated via MBAR, are in Figures S9-S11.

Because the positioning of the data points on the sketchmaps is determined only by their 13-dimensional coordinationhistogram representation, the criterion of the color coding can be varied at fixed sketch-map, to highlight different aspects of the phase-space sampling. In Figures S12-S14, we assign one color in the sketch-maps for data points belonging to the same 

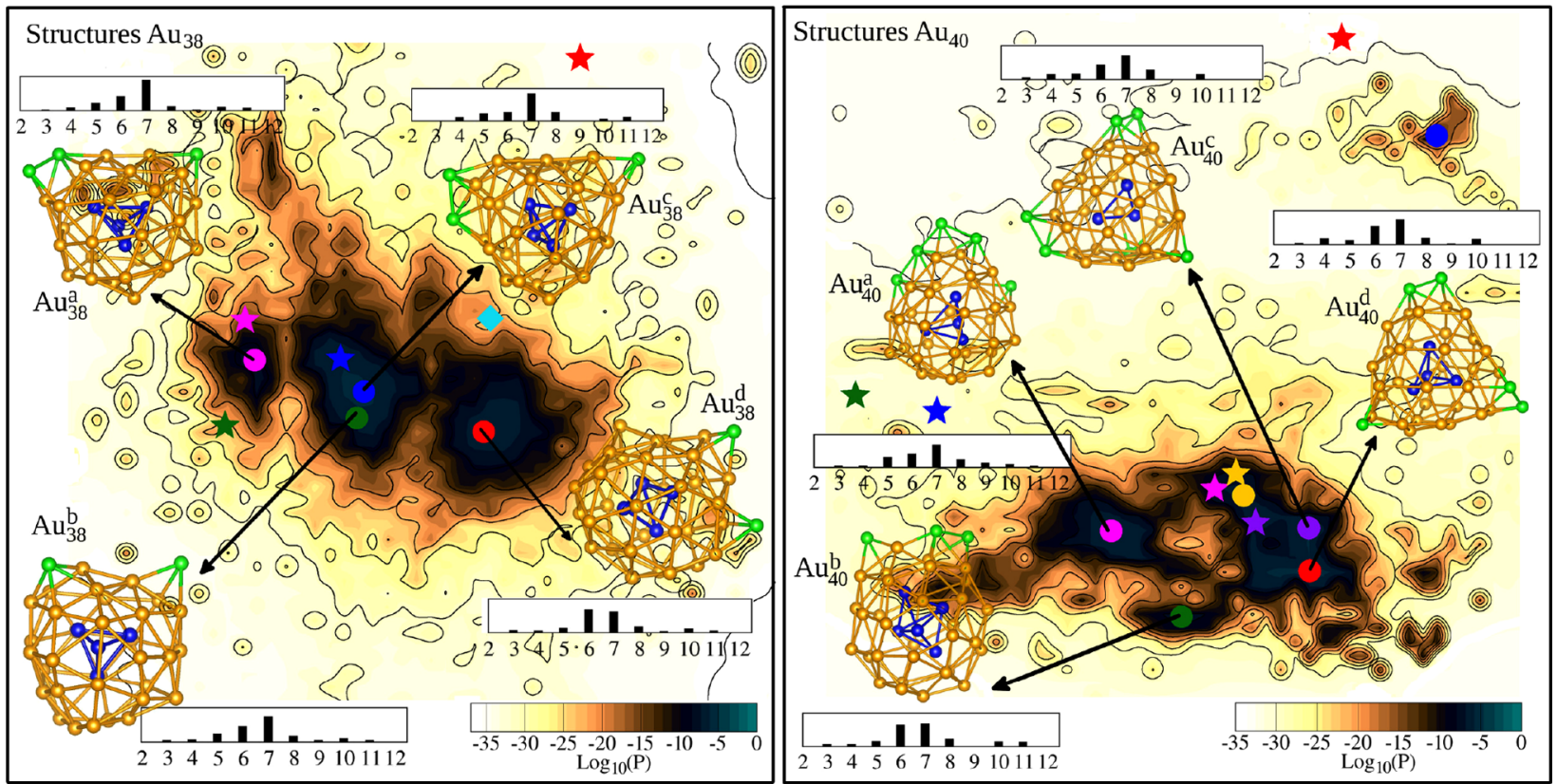

Figure 4. Probability of cluster configurations at $300 \mathrm{~K}$, calculated via MBAR as a function of the sketch-map coordinates for the population sampled for $\mathrm{Au}_{38}$ (left side, based on a total of $400 \mathrm{ps}$ of $\mathrm{MD}$ sampling) and $\mathrm{Au}_{40}$ (right side, based on 500 ps $\mathrm{MD}$ ). The selected structures distinguish core (blue), shell (yellow), and protrusion (green) regions. For each selected structure, also the corresponding 13-dimensional coordination-histogram, the input for the sketch-map dimensionality reduction, is shown. The dots mark structures selected along the MD runs, positioned in the areas of highest probability, and the stars locate the corresponding geometrically relaxed structures (dot/star of the same color indicate initial/final structures for a geometry relaxation). For $\mathrm{Au}_{38}$, the pink star is the pGMC found by us (see its structure in Figure S15). The pGMC identified in ref 19 is marked by a diamond. For $\mathrm{Au}_{40}$, the red star is the pGMC found by us, which is also the pGMC identified in ref 19.

MD trajectory, to show that the trajectories all diffuse over broad regions of the sketch-map and therefore of the configurational space.

For $\mathrm{Au}_{38}$ (Figure 4, left), we observe three rather distinct configurational basins. The structures belonging to the left and center basins have a majority of 7-fold coordinated atoms, while for the right basin, structures have a similarly high population of 6- and 7-fold coordinated atoms. The difference between the left and center basins is that structures in the center basin are more spherically shaped, while structures in the left basin are more distorted, with locally planar portions of the shell. Incidentally, both the RBHMC and "J" initial structures belong to the center basin. Furthermore, structures similar to the $\mathrm{J}$ structure are visited during the $\mathrm{MD}$ trajectories started from the RBHMC structure, suggesting a rather broad sampling of the configurational space along the $\mathrm{MD}$ trajectories. The connections between the three basins are narrow funnels, which means that, during the MD trajectories, while structures diffuse freely inside the basins, they need specific, coordinated, and infrequent structural rearrangements in order to pass from one basin to another. The power of the sketch-map is to reveal this latent order, invisible to human eye in the high-dimensional representation, even though there might not be a simple description in terms of geometrical features of the structures.

For $\mathrm{Au}_{40}$ (Figure 4, right), we also observe three basins, albeit less well-defined than for $\mathrm{Au}_{38}$. The center-bottom basin contains structures with a trigonal-bipyramidal five-atom core and a preference for 6- and 7-fold coordinated atoms. The left basin has structures with a tetrahedral four-atom core, while in the right basins there is a combination of structures with threeand four-atom cores, where the common structural motif (of the whole cluster) is the twisted trigonal pyramid. Structures in both the left- and right basins have a majority of 7-fold coordinated atoms.

For $\mathrm{Au}_{25}$ (see Figure S6), we found tubular-like structures (always with one atom in the core) with one side distorted, an aspect reminiscent of the known $\mathrm{Au}_{24}{ }^{-}$pGMC. $^{20}$ In a different basin, $\mathrm{Au}_{25}$ shows a further distortion of the tubular structure, yielding locally planar motifs.

The sketch-maps in Figure 4 (and Figure S6) report also the position of the pGMC and other local minima. We note that these structures are not located in the main basins, which means that these structures are not in the regions of the configurational space mostly visited by the MD sampling. Keeping in mind the caveats expressed above, we refer to these "darker", most visited regions as low-free-energy regions in the following. To rationalize the finding that low-energy, relaxed structures deviate significantly from the low-free-energy configurations, we selected from the MD trajectories several structures in the lowest-free-energy areas of the sketch-maps and geometrically relaxed them to find their local minimum energy structures. This is shown in Figure 4 (and Figure S6) with differently colored dots (selected structures from the MD sampling) and stars (relaxed structures), where dot-star pairs with the same color depict initial and final structures for one relaxation. In all cases, the relaxed structures are in higher-freeenergy regions compared to the selected initial structures. Interestingly, one of the selected structures from the highprobability regions, labeled as $40 \mathrm{~d}$, relaxes into the pGMC, further supporting the observation that high-probability structures at finite temperature are significantly different, based on their coordination histograms, from the pGMC as well as from the other lower-energy local minima. We inspected the change upon relaxation of (i) gyration radius, (ii) number of core-shell bonds, and (iii) the average effective 
coordination number ${ }^{43,44}(\mathrm{ECN})$. The ECN is an indicator of the average coordination of atoms in a cluster. It adopts a selfconsistent, parameter-free, smoothly decaying definition of bond and therefore coordination number for each atom. These values are reported in Table S3. As a general trend, the gyration radius decreases and the number of core-shell bonds increases upon relaxation. At the same time, the ECN always increases. This indicates that there is a contraction of the clusters upon relaxation and, in particular, a tightening of the core-shell binding. Together with the bond lifetime analysis (Figure 3), this observation further strengthens the picture of typical gold cluster structures at $300 \mathrm{~K}$ and higher temperatures with weak coupling between core and shell and therefore larger configurational freedom (hence lower free energy) than relaxed structures.

An analysis of the dynamics of the single trajectories projected onto the 2-D maps reveals that each trajectory of $\mathrm{Au}_{38}$ and $\mathrm{Au}_{40}$ visits along its 50 ps all the main basins in the map (i.e., those with an exemplary structure shown in the plots), swapping between neighboring basins between 10 and 100 times. Much slower is the diffusion for $\mathrm{Au}_{25}$, where at 300 $\mathrm{K}$ the trajectories never leave the basin they start in, but in all three cases, during the initial 50 ps at higher temperature, the trajectory swaps few times between the two main basins. We can therefore conclude that, although full ergodicity of our trajectories cannot be ensured and indeed we notice signatures of some kinetic trapping, single trajectories do not merely (anharmonically) vibrate in one basin but their diffusion across the 2-D maps is significant.

We note, incidentally, that the relaxation of the low-freeenergy structures let us identify pGMC for $\mathrm{Au}_{25}$ and $\mathrm{Au}_{38}$, which are previously unreported in the literature (pink stars in Figures 4 (left) and S6). The corresponding structures are shown in Figure S15. For $\mathrm{Au}_{40}$, the pGMC we found (red star in Figure 4 (right)) corresponds with the pGMC in ref 19.

Finally, we investigated the reason for the stability of threedimensional four- and five-atom cores, while isolated neutral $\mathrm{Au}_{4}$ and $\mathrm{Au}_{5}$ are known to be planar. ${ }^{28}$ We measured the amount of positive charge needed to stabilize the 3-D structure versus the planar one for both $\mathrm{Au}_{4}$ and $\mathrm{Au}_{5}$. In Figure 5, we

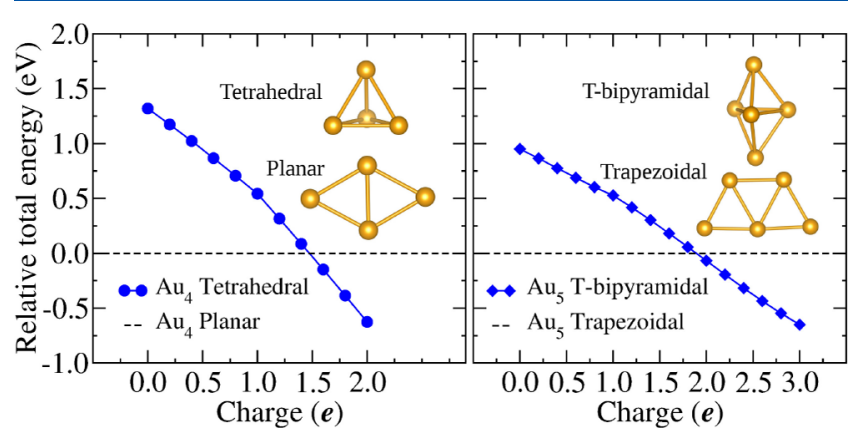

Figure 5. Left (right): Relative stability of the tetrahedral (trigonalbipyramidal) structure with respect to the planar $\mathrm{Au}_{4}\left(\mathrm{Au}_{5}\right)$ structure, as a function of the positive total charge of the cluster.

show the energy of the 3-D structure, relative to the planar one for both $\mathrm{Au}_{4}$ and $\mathrm{Au}_{5}$, where both structures are geometrically unrelaxed for each charge. Some structures like the neutral tetrahedron or trigonal-bipyramidal are mechanically unstable, i.e., have imaginary vibrational frequencies (see Figures S16 and S17). In Figure S16, we show the relaxation of the structures by fixing their symmetry and in Figure S17 without fixing symmetry. We find that tetrahedral $\mathrm{Au}_{4}$ becomes more stable than planar $\mathrm{Au}_{4}$ with a charge of $1.5 e$, while trigonalbipyramidal $\mathrm{Au}_{5}$ needs $2.0 e$ to become more stable than planar $\mathrm{Au}_{5}$. These charge values, even without symmetry constraints, are much larger than the small positive charges observed for the core atoms. Therefore, charge alone is not sufficient to stabilize the 3-D structures, leaving confinement and bonding interactions with the shell as likely reasons for the stabilization.

In summary, we have presented a theoretical study of the stability and dynamics of $\mathrm{Au}_{25}, \mathrm{Au}_{38}$, and $\mathrm{Au}_{40}$ nanoclusters whose configurational space is sampled via Born-Oppenheimer molecular dynamics at various temperatures. We showed that in the considered range of temperatures (between 300 and $600 \mathrm{~K}$ ) the gold clusters are not amorphous. Instead, they exhibit a dynamical core-shell structure, with a cationic core (single $\mathrm{Au}$ for $\mathrm{Au}_{25}$ and mainly tetrahedral $\mathrm{Au}_{4}$ for $\mathrm{Au}_{38}$ and $\mathrm{Au}_{40}$ ) loosely bound to an outer anionic flexible shell. We also identify, as a persistent feature, a substructure in the cluster shells- one triangular-lattice region with 6- and 7-fold coordinated atoms and an outer region with fewer lowercoordinated atoms that we term protrusions. By using sketchmaps-two-dimensional embeddings of the coordinationhistogram representation of the clusters-and Boltzmann reweighting, we built approximate free-energy landscapes, at room temperature, of the studied nanoclusters. These show that the global and local minimum energy structures differ significantly from the structures in low free-energy areas, i.e., the most probable at finite temperature. Obtained by using a suite of statistical tools that can be applied readily to similar systems, our results are able to highlight some typical characteristics of the finite-temperature populations of $\mathrm{Au}_{25}$, $\mathrm{Au}_{38}$, and $\mathrm{Au}_{40}$, providing insights into the stability and dynamics of their core-shell regions. Our results (especially those for $\mathrm{Au}_{38}$ and $\mathrm{Au}_{40}$ ) suggest a possible mechanism of (equilibrium) cluster growth. If gold atoms are added to a nanocluster in the size range that we have considered, they will tend to remain in the shell and protrusion regions until a critical value is reached when one of the gold atoms stably transfers to the core.

\section{ASSOCIATED CONTENT}

\section{S Supporting Information}

The Supporting Information is available free of charge on the ACS Publications website at DOI: 10.1021/acs.jpclett.8b03397.

Detailed computational details for the EAM-RBHMC, DFT-MD, and sketch-map-training calculations; the $g(r)$ and Hirshfeld analyses for all MD trajectories; the resulting sketch-map for $\mathrm{Au}_{25}$; MBAR-estimated error bars on the probabilities reported in all the sketch-maps; detailed bond-lifetime analysis for each trajectory; detailed information on all found local minima; and further information on stability analysis of charged $\mathrm{Au}_{4}$ and $\mathrm{Au}_{5}$ (PDF)

\section{AUTHOR INFORMATION}

\section{Corresponding Authors}

*E-mail: guedes.sobrinho.d@gmail.com.

*E-mail: ghiringhelli@fhi-berlin.mpg.de.

ORCID

Diego Guedes-Sobrinho: 0000-0002-3313-2822

Juarez L. F. Da Silva: 0000-0003-0645-8760 
Luca M. Ghiringhelli: 0000-0001-5099-3029

\section{Notes}

The authors declare no competing financial interest. All the MD-trajectory and geometry-relaxation files are uploaded in the NOMAD Repository, https://repository. nomad-coe.eu/NomadRepository-1.1. See the Supporting Information for further details on how to locate this data.

\section{ACKNOWLEDGMENTS}

We thank Matthias Scheffler for his support and Bryan Goldsmith for a critical reading of the manuscript and insightful suggestions. D.G.-S. and J.L.F.D.S. acknowledge support from FAPESP (São Paulo Research Foundation, Grant Numbers 2017/11631-2 and 2014/22044-2), Shell, and the strategic importance of the support given by ANP (Brazils National Oil, Natural Gas and Biofuels Agency) through the R\&D levy regulation. D.G.-S. and J.L.F.D.S. also thank the National Council for Scientific and Technological Development $(\mathrm{CNPq})$ and the Coordination for Improvement of Higher Level Education (CAPES) for financial support. I.H. thanks the National Sciences and Engineering Research Council (NSERC) for financial support. L.M.G. acknowledges for financial support the European Union Horizon 2020 research and innovation program (\#676580: The NOMAD Laboratory-an European Center of Excellence and \#740233: TEC 1p) and the Berlin Big-Data Center (BBDC, \#01IS14013E). Computer time was provided by the Max Planck Computing and Data Facility, the Laboratory of Advanced Scientific Computing (University of São Paulo), the Department of Information Technology-Campus São Carlos, and Compute Canada.

\section{REFERENCES}

(1) III Aiken, J. D.; Finke, R. G. A. A Review of Modern TransitionMetal Nanoclusters: Their Synthesis, Characterization, and Applications in Catalysis. J. Mol. Catal. A: Chem. 1999, 145, 1-44.

(2) Jin, R. Atomically Precise Metal Nanoclusters: Stable Sizes and Optical Properties. Nanoscale 2015, 7, 1549-1565.

(3) Marjomäki, V.; Lahtinen, T.; Martikainen, M.; Koivisto, J.; Malola, S.; Salorinne, K.; Pettersson, M.; Häkkinen, H. Site-Specific Targeting of Enterovirus Capsid by Functionalized Monodisperse Gold Nanoclusters. Proc. Natl. Acad. Sci. U. S. A. 2014, 111, 12771281.

(4) Chaves, A. S.; Piotrowski, M. J.; Da Silva, J. L. F. Structural, Energetics, and Electronic Properties Evolution of the $3 d, 4 d$, and $5 d$ Transition-Metal Clusters $\left(30 \mathrm{TM}_{\mathrm{n}}\right.$ Systems for $\left.\mathrm{n}=2-15\right)$ : A Density Functional Theory Investigation. Phys. Chem. Chem. Phys. 2017, 19, 15484-15502.

(5) Mostafa, S.; Behafarid, F.; Croy, J. R.; Ono, L. K.; Li, L.; Yang, J. C.; Frenkel, A. I.; Cuenya, B. R. Shape-Dependent Catalytic Properties of Pt Nanoparticles. J. Am. Chem. Soc. 2010, 132, 15714-15719.

(6) Chaves, A. S.; Rondina, G. G.; Piotrowski, M. J.; Tereshchuk, P.; Da Silva, J. L. F. The Role of Charge States in the Atomic Structure of Cun and Ptn ( $\mathrm{n}=2-14$ Atoms) Clusters: A DFT Investigation. J. Phys. Chem. A 2014, 118, 10813-10821.

(7) Jadzinsky, P. D.; Calero, G.; Ackerson, C. J.; Bushnell, D. A.; Kornberg, R. D. Structure of a Thiol Monolayer-Protected Gold Nanoparticle at 1.1 A Resolution. Science 2007, 318, 430-433.

(8) Yamauchi, H.; Ito, S.; Yoshida, K.-i.; Itoh, T.; Tsuboi, Y.; Kitamura, N.; Miyasaka, H. Temperature Near Gold Nanoparticles under Photoexcitation: Evaluation Using a Fluorescence Correlation Technique. J. Phys. Chem. C 2013, 117, 8388-8396.

(9) Sugimura, Y.; Suzuki, Y. A. A High-Temperature Catalytic Oxidation Method for the Determination of Non-Volatile Dissolved
Organic Carbon in Seawater by Direct Injection of a Liquid Sample. Mar. Chem. 1988, 24, 105-131.

(10) Haruta, M. When Gold Is Not Noble: Catalysis by Nanoparticles. Chem. Rec. 2003, 3, 75-87.

(11) Häkkinen, H.; Barnett, R. N.; Landman, U. Electronic Structure of Passivated Au38 (SCH3)24 Nanocrystal. Phys. Rev. Lett. 1999, 82, 3264-3267.

(12) Heaven, M. W.; Dass, A.; White, P. S.; Holt, K. M.; Murray, R. W. Crystal Structure of the Gold Nanoparticle [N(C8 H17)4][Au25 (SCH2 CH2 Ph)18]. J. Am. Chem. Soc. 2008, 130, 3754-3755.

(13) Farrag, M.; Tschurl, M.; Dass, A.; Heiz, U. Infra-Red Spectroscopy of Size Selected Au25, Au38 and Au144 Ligand Protected Gold Clusters. Phys. Chem. Chem. Phys. 2013, 15, 12539.

(14) Azubel, M.; Koivisto, J.; Malola, S.; Bushnell, D.; Hura, G. L.; Koh, A. L.; Tsunoyama, H.; Tsukuda, T.; Pettersson, M.; Häkkinen, H.; Kornberg, R. D. Electron Microscopy of Gold Nanoparticles at Atomic Resolution. Science 2014, 345, 909-912.

(15) Jin, R.; Zeng, C.; Zhou, M.; Chen, Y. Atomically Precise Colloidal Metal Nanoclusters and Nanoparticles: Fundamentals and Opportunities. Chem. Rev. 2016, 116, 10346-10413.

(16) Garzón, I. L.; Beltrán, M. R.; González, G.; Gutierrez-González, I.; Michaelian, K.; Reyes-Nava, J. A.; Rodriguez-Hernández, J. I. Chirality, defects, and disorder in gold clusters. Eur. Phys. J. D 2003, 24, 105-109.

(17) Xing, X.; Yoon, B.; Landman, U.; Parks, J. H. Structural Evolution of $\mathrm{Au}$ Nanoclusters: From Planar to Cage to Tubular Motifs. Phys. Rev. B: Condens. Matter Mater. Phys. 2006, 74, 165423.

(18) Bulusu, S.; Li, X.; Wang, L.-S.; Zeng, X. C. Structural Transitions from Pyramidal to Fused Planar to Tubular to Core/Shell Compact in Gold Clusters: $\mathrm{Au}_{n}(\mathrm{n}=21-25)$. J. Phys. Chem. C 2007, $111,4190-4198$

(19) Jiang, D.; Walter, M. $A_{40}$ : A Large Tetrahedral Magic Cluster. Phys. Rev. B: Condens. Matter Mater. Phys. 2011, 84, 193402.

(20) Wang, L.; Wang, L. Probing the Electronic Properties and Structural Evolution of Anionic Gold Clusters in the Gas Phase. Nanoscale 2012, 4, 4038.

(21) Zeng, C.; Chen, Y.; Das, A.; Jin, R. Transformation Chemistry of Gold Nanoclusters: From One Stable Size to Another. J. Phys. Chem. Lett. 2015, 6, 2976-2986.

(22) Shao, N.; Huang, W.; Mei, W.-N.; Wang, L. S.; Wu, Q.; Zeng, X. C. Structural Evolution of Medium-Sized Gold Clusters $\mathrm{Au}_{\mathrm{n}}-(\mathrm{n}=$ 36, 37, 38): Appearance of Bulk-Like Face Centered Cubic Fragment. J. Phys. Chem. C 2014, 118, 6887-6892.

(23) Pande, S.; Huang, W.; Shao, N.; Wang, L.; Khetrapal, N.; Mei, W.; Jian, T.; Wang, L.; Zeng, X. C. Structural Evolution of Core-Shell Gold Nanoclusters: $\mathrm{Au}_{\mathrm{n}}{ }^{-}(\mathrm{n}=42-50)$. ACS Nano 2016, 10, 1001310022.

(24) Cleveland, C. L.; Luedtke, W. D.; Landman, U. Melting of Gold Clusters. Phys. Rev. B: Condens. Matter Mater. Phys. 1999, 60, 50655077.

(25) de Bas, B. S.; Ford, M. J.; Cortie, M. B. Melting in Small Gold Clusters: a Density Functional Molecular Dynamics Study. J. Phys.: Condens. Matter 2006, 18, 55-74.

(26) Kang, J.; Wei, S.; Kim, Y. Origin of the Diverse Melting Behaviors of Intermediate-Size Nanoclusters: Theoretical Study of AlN (N = 51-58, 64). J. Am. Chem. Soc. 2010, 132, 18287-18291.

(27) Beret, E. C.; Ghiringhelli, L. M.; Scheffler, M. Free Gold Clusters: Beyond the Static, Monostructure Description. Faraday Discuss. 2011, 152, 153.

(28) Ghiringhelli, L. M.; Gruene, P.; Lyon, J. T.; Rayner, D. M.; Meijer, G.; Fielicke, A.; Scheffler, M. Not so Loosely Bound Rare Gas Atoms: Finite-Temperature Vibrational Fingerprints of Neutral GoldCluster Complexes. New J. Phys. 2013, 15, No. 083003.

(29) Perdew, J. P.; Burke, K.; Ernzerhof, M. Generalized Gradient Approximation Made Simple. Phys. Rev. Lett. 1996, 77, 3865-3868.

(30) Tkatchenko, A.; Ambrosetti, A.; DiStasio, R. A. Interatomic Methods for the Dispersion Energy Derived from the Adiabatic Connection Fluctuation-Dissipation Theorem. J. Chem. Phys. 2013, 138, No. 074106 
(31) Blum, V.; Gehrke, R.; Hanke, F.; Havu, P.; Havu, V.; Ren, X.; Reuter, K.; Scheffler, M. Ab Initio Molecular Simulations with Numeric Atom-Centered Orbitals. Comput. Phys. Commun. 2009, 180, 2175-2196.

(32) Bussi, G.; Donadio, D.; Parrinello, M. Canonical Sampling Through Velocity Rescaling. J. Chem. Phys. 2007, 126, No. 014101.

(33) Yu, C.; Harbich, W.; Sementa, L.; Ghiringhelli, L. M.; Aprá, E.; Stener, M.; Fortunelli, A.; Brune, H. Intense Fluorescence of $\mathrm{Au}_{20}$. J. Chem. Phys. 2017, 147, No. 074301.

(34) Häkkinen, H.; Moseler, M.; Kostko, O.; Morgner, N.; Hoffmann, M. A.; v. Issendorff, B. Symmetry and Electronic Structure of Noble-Metal Nanoparticles and the Role of Relativity. Phys. Rev. Lett. 2004, 93, 093401.

(35) Huang, W.; Ji, M.; Dong, C.-D.; Gu, X.; Wang, L.-M.; Gong, X. G.; Wang, L. Relativistic Effects and the Unique Low-Symmetry Structures of Gold Nanoclusters. ACS Nano 2008, 2, 897-904.

(36) Van Lenthe, J. H.; Faas, S.; Snijders, J. G. Gradients in the Ab Initio Scalar Zeroth-Order Regular Approximation (ZORA) Approach. Chem. Phys. Lett. 2000, 328, 107-112.

(37) Rondina, G. G.; Da Silva, J. L. F. A Revised Basin-Hopping Monte Carlo Algorithm for Structure Optimization of Clusters and Nanoparticles. J. Chem. Inf. Model. 2013, 53, 2282-2298.

(38) Vargas, A.; Santarossa, G.; Iannuzzi, M.; Baiker, A. Fluxionality of Gold Nanoparticles Investigated by Born-Oppenheimer Molecular Dynamics. Phys. Rev. B: Condens. Matter Mater. Phys. 2009, 80, 195421.

(39) Hirshfeld, F. L. Bonded-Atom Fragments for Describing Molecular Charge Densities. Theoret. Chim. Acta 1977, 44, 129-138.

(40) Ceriotti, M.; Tribello, G. A.; Parrinello, M. Simplifying the Representation of Complex Free-energy Landscapes Using Sketchmap. Proc. Natl. Acad. Sci. U. S. A. 2011, 108, 13023-13028.

(41) Ceriotti, M.; Tribello, G. A.; Parrinello, M. Demonstrating the Transferability and the Descriptive Power of Sketch-map. J. Chem. Theory Comput. 2013, 9, 1521-1532.

(42) Shirts, M. R.; Chodera, J. D. Statistically Optimal Analysis of Samples from Multiple Equilibrium States. J. Chem. Phys. 2008, 129, 124105.

(43) Hoppe, R. The Coordination Number - an "Inorganic Chameleon. Angew. Chem., Int. Ed. Engl. 1970, 9, 25-34.

(44) Da Silva, J. L. F. Effective Coordination Concept Applied for Phase Change $(\mathrm{GeTe})_{m}\left(\mathrm{Sb}_{2} \mathrm{Te}_{3}\right)_{n}$ Compounds. J. Appl. Phys. 2011, 109, No. 023502. 